\title{
Creatine Kinase
}

National Cancer Institute

\section{Source}

National Cancer Institute. Creatine Kinase. NCI Thesaurus. Code C113245.

An enzyme complex that can reversibly convert ATP and creatine to phosphocreatine and ADP. Cytosolic creatine kinases are comprised of homodimers or heterodimers of creatine kinase B-type protein and creatine kinase M-type protein. Mitochondrial creatine kinases are octomers comprised of either four homodimers of creatine kinase U-type, mitochondrial protein or four creatine kinase S-type, mitochondrial protein homodimers. 